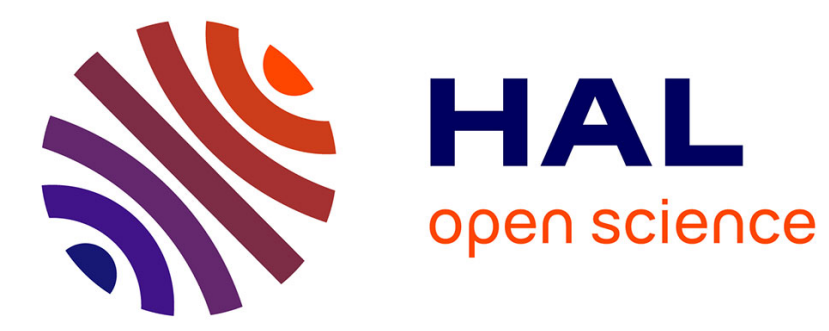

\title{
Modeling, Control and Simulation of Upward Jump of a Biped
}

\author{
Yannick Aoustin
}

\section{To cite this version:}

Yannick Aoustin. Modeling, Control and Simulation of Upward Jump of a Biped. Multi, 2012, pp.1-21. 10.1007/s11044-012-9319-6 . hal-00718175

\section{HAL Id: hal-00718175 \\ https://hal.science/hal-00718175}

Submitted on 16 Jul 2012

HAL is a multi-disciplinary open access archive for the deposit and dissemination of scientific research documents, whether they are published or not. The documents may come from teaching and research institutions in France or abroad, or from public or private research centers.
L'archive ouverte pluridisciplinaire HAL, est destinée au dépôt et à la diffusion de documents scientifiques de niveau recherche, publiés ou non, émanant des établissements d'enseignement et de recherche français ou étrangers, des laboratoires publics ou privés. 


\title{
Modeling, Control and Simulation of Upward Jump of a Biped
}

\author{
Y. Aoustin \\ LUNAM, Institut de Recherche en \\ Communications et Cybernétique de Nantes, \\ UMR 6597, \\ CNRS, École Centrale de Nantes, Université \\ de Nantes, France. \\ A. M. Formalskii \\ Institute of Mechanics, Lomonosov Moscow \\ State University.
}

the date of receipt and acceptance should be inserted later

\begin{abstract}
This paper explores the vertical upward jumping of a planar biped. The jumping process is decomposed into the crouching phase, the thrust in the knees, the flight phase, the touchdown and the straightening up movement of the biped. A mathematical model for this kind of jump of the biped is developed. Torques are applied in the hip and knee joints. The degree of underactuation of the mechanism is equal to one in the support phase and to three in the flight phase. The control algorithm is designed to ensure the jump of the biped. This algorithm is such that the center of mass of the mechanism is always placed on the same vertical line. The biped touches the ground in the same place where it starts from. The synthesis of the jumping process is supported by simulations which give consistent results with human data from existing biomechanical literature. Furthermore, the stick diagram of the jump derived from these simulation results seems natural for the human jumping. The problem of energy recovery is considered for the jumping of the biped by using springs in the hip and knee joints. The springs have an influence to minimize the mechanical energy consumed by the drives in the hip and knee joints. The springs in the knees help to increase the lifting of the bipedal mechanism.
\end{abstract}

Keywords: Biped; Degree of underactuation; Constraints; Crouching phase; Flight phase; Touchdown; Inelastic impact; Energy consumption.

Corresponding author: Address: 1, rue de la Noë, BP 92101. 44321 Nantes, France. (e-mail: Yannick.Aoustin@irccyn.ec-nantes.fr).

Address: 1, Michurinskii Prospect, Moscow, 119192, Russia. (e-mail: formal@imec.msu.ru). This work was supported by Russian Foundation for Basic Research, Grant 07-01-92167 and CNRS via a Project of International Collaboration Scientific, PICS 3866. 


\section{Introduction}

In the last 30 years many research efforts in the field of mobile robots have been devoted to the development of bipedal robots and humanoid robots acting in a daily life. Many research efforts have been devoted to study the human locomotion also. Bipeds have unilateral constraints with the ground. Depending on their adopted gaits, bipedal robots can be underactuated, fullactuated or overactuated mechanisms. Their autonomy in energy, power of their actuators is limited. Despite these difficulties, one of the most important objectives is to approach the human's performances as close as possible. Many results have been established about static stable walking gaits [1], [35], dynamical stable walking gaits [16], [7], [32], [3], passive-dynamic walking gaits, [14], [24], [10], [36] and running gaits, [31], [5], [8], [11].

Many works are also devoted to the jumping, with which this article deals. The three-dimensional (3D) mechanism, which hops and runs on one springy leg has been studied theoretically and experimentally by Raibert et al., [30]. In [18], Itiki et al. present a study of vertical jumping through a biomechanical model of the leg. Meghdari and Aryanpour [25] proposed a dynamical model for the human jumping process. This model uses dynamic relations to compute the driving torques in the joints of the human body. An integrated motion control method related to a bipedal humanoid walk, jump and run is applied to the real humanoid "QRIO" by Nagasaka et al., [26]. Zajac et al. show in [40] that compliant tendons and calf muscles that are fast and strong are essential for humans to jump as high as possible using only the ankles for propulsion. In robotics, it would be a manner to increase the mobility of humanoid robots to avoid an obstacle, for running. For example Kajita and his colleagues introduce the bipedal robot HRP-2LR and its hopping with both legs as the first attempt towards running [20]. Using data obtained from human jumping phases, Sakka and Yokoi [33] define a humanoid reference jumping trajectory which is as close as possible, under predefined constraints, to a human jump which is derived from the ground reaction forces using an arm-swing. Landing stability of jumping gaits is studied for a fourlink planar biped model in [15]. Consider also this example about a quadruped robot Semiquad, where a jump on the front leg (respectively the rear leg) allows it to adopt a curvet walking gait [3]. For the take-off phase of a sub-optimal long jump several criteria are evaluated with the comparison of the obtained movements through a parametric optimization with an actual performance [19]. This paper aims to identify the criterion which most closely approximated that spontaneously minimized by the athlete. An actual jumping motion is used to define the local joint-constraint model for the shoulder joint in [12].

From biomechanical data Ker et al. [21] observed that, during the walking $78 \%$ of the storage energy by the compliant elements of the stance foot are restored. Then, one natural way to minimize a consumed mechanical energy is to equip the biped with springs or with elastic actuators, see [28], [39], [38], [29], [37] or [34]. The development of bipedal robots WL-14 [39], [38], [13] and Lucy [37] shows that the presence of the variable stiffness in their drives reduces the transportation cost by tuning the drive mechanism stiffness. Farrell et al. [13] show that optimizing the motion and the stiffness of the springs located in the ankle of a five-link planar biped results in an energy efficient walking. A bipedal robot with knees, which have a revolute series compliant actuator is presented in [17]. Many problems are still open however, especially for the fast dynamics during the jump. There is a lack of sound dynamic models for the complete jumping 
process with a phase to prepare the flight, a flight phase, a touchdown and so on. Vertical jumping with minimal mechanical energy is still an object of investigation.

Contribution. To modelize the human jump, it is necessary to choose a biped model, which is at the same time relatively simple to define a rigorous statement of the problem and relatively close to human in order to evaluate the most important physical phenomena. A compromise with a simple dynamic model of a planar biped composed of a one-link trunk and two identical two-link legs with knees is proposed to study the vertical jump. The degree of underactuation for the biped is one on the support phase and equals three in the flight phase. The control algorithm to organize the jump is developed. To the best of our knowledge there is not any similar model and control algorithm in the literature. To prevent a horizontal displacement of the center of mass of the biped the constraint is introduced in the model. After crouching of the biped, a constant thrust torque in open loop is applied in the knees to cancel (after some time) the ground reaction in the leg tips and then to obtain the flight phase. The jump height is managed by this thrust torque. For the touchdown an absolutely inelastic impact is assumed. For the jumping of human the ground reaction is large at the time of the touchdown. Here this ground reaction is described by a Dirac delta-function. However in reality for the jumping of human the ground reaction is bounded. Therefore, our model can be considered as an asymptotical model. Springs are added in the knees and hips to make the vertical jumping easier. Simulation results are proposed to analyze this vertical jumping and they are consistent with biomechanical data given in the existing literature. As far as energy consumption is concerned, numerical investigation shows that for a given lifting of the biped, there is an optimal choice for the springs in the knees and hips. But the influence of the spring to preserve energy is not large.

From our perspective this study is interesting for the modeling of the human jump and for the design of a jumping bipedal robot.

This paper is organized as follows. Section 2 describes the methodology of the modeling of the biped jump. This Section consists of several Subsections. In Subsection 2.1 we present the biped mechanism. Subsections 2.2 and 2.3 contain the dynamic and impact models. Control algorithm to obtain the vertical jumping is presented in Subsection 2.4. Subsection 2.5 is devoted to the problem of energy recovery using springs in the joints. The physical parameters of the biped used in simulation are described in Subsection 2.6. In Section 3 we describe the results of simulation. We discuss our simulation results and compare them with experimental data in Section 4. Section 5 offers our conclusions and perspectives.

\section{Methods}

In this section, we present the methodology of the mathematical modeling of the biped jump. This methodology depends on the interaction with the ground and the degree of underactuation for the different phases of the biped jump.

\subsection{Biped Presentation}

The object of our study is a three-link planar biped mechanism. The double takeoff of this biped is studied. Assume that both shins are identical and coupled (see link $a b$ in Figure 1), both thighs are also identical and coupled (see link $b c$ ). The trunk is 
depicted in Figure 1 by link $c d$. Let link $a b$ be the first, link $b c$ - the second and link $c d$ - the third. Let us denote by $l_{i}(i=1,2$ and 3$)$ the lengths of the first, second and third link, by $m_{i}$ the masses of these links. Let $I_{i}(i=1,2$ and 3$)$ be the inertia moment of the $i$-th link about its center of mass, $s_{i}$ be the distances between the center of mass of the $i$-th link and joints $a, b$ and $c . M=m_{1}+m_{2}+m_{3}$ is the global mass of the biped.

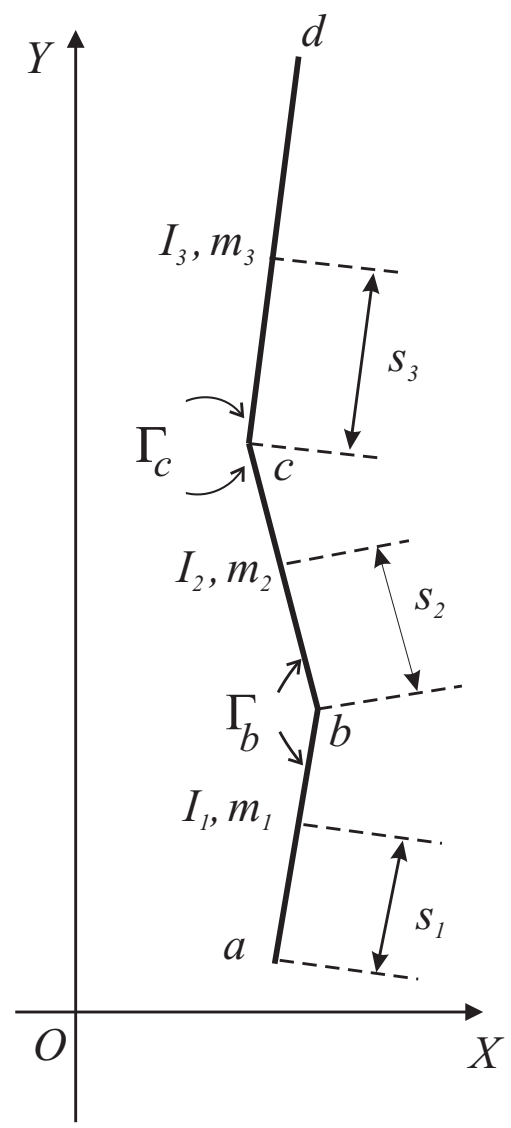

Fig. 1 The three-link biped in the flight phase.

Two phases of the jumping are considered: a support phase when the leg tips are in contact with the ground and a flight phase when the leg tips do not have any contact. In support phase, the vertical component of the ground reaction must be directed upwards. Flight phase starts when the ground reaction becomes zero. After the flight phase the touchdown occurs with an impact and a new support phase starts. During support phase, our system has three degrees of freedom. In the flight phase our biped has five degrees of freedom.

Torques $\Gamma_{b}$ and $\Gamma_{c}$ can be applied in the knee and hip joints respectively (see Figure 1). Torques in the ankle joints $a$ are always absent (equal to zero). The simulation 
of the three-link model (without feet) simplifies the transitions between the support phase and the flight phase.

\subsection{Planar Biped Model}

In order to introduce the generalized coordinates of our system, we consider the following diagram of the three-link mechanism, Figure 2.

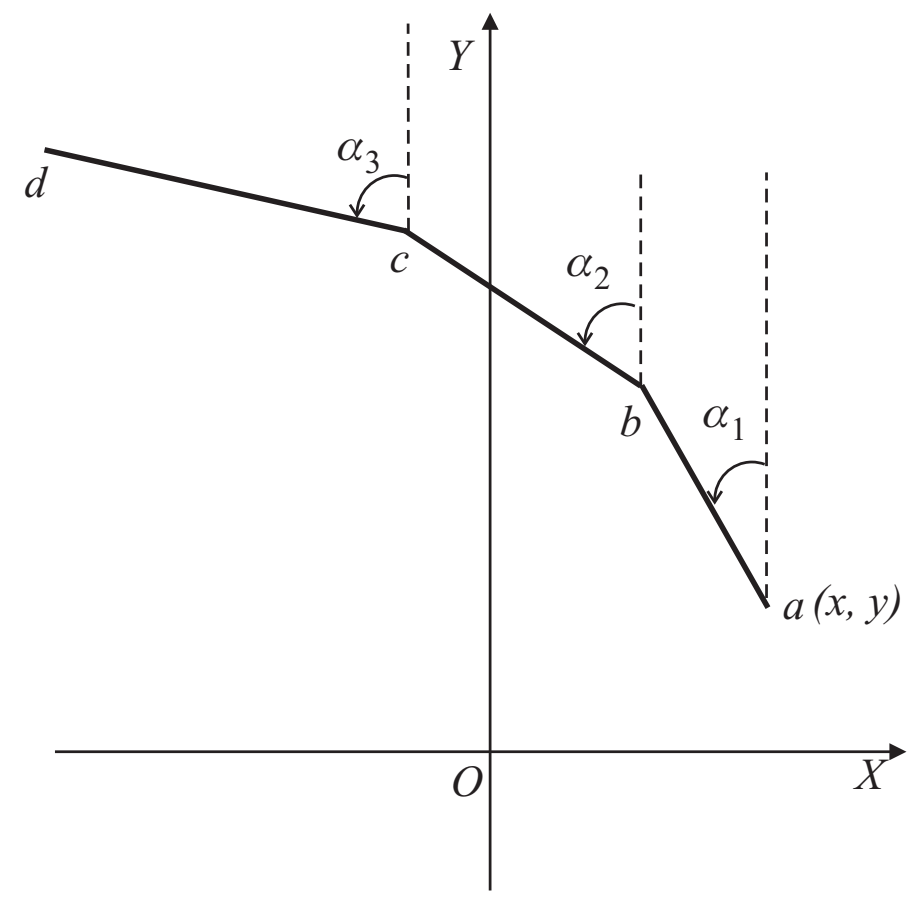

Fig. 2 Generalized coordinates of the biped.

The Cartesian coordinates of the leg tips are denoted by $x$ and $y$. The variables $\alpha_{1}, \alpha_{2}$, and $\alpha_{3}$ are the angles between the vertical line and the first $a b$, second $b c$, and third $c d$ link respectively. Let $\alpha=\left[\alpha_{1}, \alpha_{2}, \alpha_{3}\right]^{\mathrm{t}}$ be ${ }^{1}$.

Using the second Lagrange method, we develop the equations of motion during the flight phase in the following compact matrix form:

$$
\mathbf{D}(\alpha) \ddot{\mathbf{q}}+\mathbf{C}(\alpha)\left[\dot{q}_{i}^{2}\right]+g \mathbf{E}\left[\sin q_{i}\right]=\left[\begin{array}{rr}
1 & 0 \\
-1 & 1 \\
0 & -1 \\
0 & 0 \\
0 & 0
\end{array}\right]\left[\begin{array}{l}
\Gamma_{b} \\
\Gamma_{c}
\end{array}\right]
$$

\footnotetext{
1 Here symbol "t" denotes transposition
} 
Here we denote

$$
\mathbf{q}=\left[\begin{array}{c}
\alpha_{1} \\
\alpha_{2} \\
\alpha_{3} \\
x \\
y
\end{array}\right]=\left[\begin{array}{c}
\alpha \\
x \\
y
\end{array}\right],\left[\dot{q}_{i}^{2}\right]=\left[\begin{array}{c}
\dot{\alpha}_{1}^{2} \\
\dot{\alpha}_{2}^{2} \\
\dot{\alpha}_{3}^{2} \\
0 \\
0
\end{array}\right],\left[\sin q_{i}\right]=\left[\begin{array}{c}
\sin \alpha_{1} \\
\sin \alpha_{2} \\
\sin \alpha_{3} \\
0 \\
1
\end{array}\right]
$$

Matrices $\mathbf{D}(\alpha), \mathbf{C}(\alpha)$ and $\mathbf{E}$, of size $(5 \times 5)$, are presented in Appendix. Inertia matrix $\mathbf{D}(\alpha)$ is definite positive and symmetrical.

Motion of our biped in support phase, with point $a$ on the ground, is governed by:

$$
\mathbf{D}(\alpha) \ddot{\mathbf{q}}+\mathbf{C}(\alpha)\left[\dot{q}_{i}^{2}\right]+g \mathbf{E}\left[\sin q_{i}\right]=\left[\begin{array}{rr}
1 & 0 \\
-1 & 1 \\
0 & -1 \\
0 & 0 \\
0 & 0
\end{array}\right]\left[\begin{array}{l}
\Gamma_{b} \\
\Gamma_{c}
\end{array}\right]+\left[\begin{array}{ll}
0 & 0 \\
0 & 0 \\
0 & 0 \\
1 & 0 \\
0 & 1
\end{array}\right]\left[\begin{array}{l}
R_{x} \\
R_{y}
\end{array}\right]
$$

Here $R_{x}$ and $R_{y}$ are the horizontal and vertical components of the ground reaction $\mathbf{R}$ applied in point $a$ respectively.

Of course we can choose the relative angle between links $a b$ and $b c$, the relative angle between links $b c$ and $c d$ as generalized coordinates, instead of the absolute angles $\alpha_{2}$ and $\alpha_{3}$. But using these absolute angles $\alpha_{2}$ and $\alpha_{3}$ as generalized coordinates, the mathematical model is more illustrative and understandable for the considered problem. The absolute angles are used as generalized coordinates in [14] to develop the mathematical model of a walking biped and in [22] to consider the problem of jumping. So, it seems that using absolute angles as generalized coordinates is more convenient from a methodical point of view.

Before the takeoff in the human vertical jump and after landing the feet usually do not move in the horizontal direction. Then we can consider that the coefficient of the friction is high enough to avoid to slide in support. (Pandy and Zajac [27] observed experimentally and by simulation that before to jump the maximum amplitude of the biphasic horizontal ground reaction represents near $30 \%$ of body weight. The maximum amplitude of the vertical ground reaction represents $200 \%$ of body weight). Therefore, we assume that in the support phase the biped never slides on the ground and the abscissa of its leg tips is constant. Let this constant be zero.

$$
x=0
$$

The $y$-coordinate of the leg tips is also zero in support phase

$$
y=0
$$

After the vertical jump (after landing) human comes back to the ground at the same place approximately. Therefore, looking ahead we note that the control strategy is designed in this paper to satisfy equality (4) also in the flight phase.

Pandy and Zajac [27] show also that before to jump the value of the horizontal component of the ground reaction is close to zero upon the average. After landing and 
the straightening up movement of human the position of its center of mass becomes equal to initial one (approximately). It means that in the jumping process an "average" value of the horizontal component of the ground reaction is close to zero. Therefore, for simplification we will design the control for our biped with the following constraint: abscissa $x_{G}$ of the center of mass $G$ of the biped is always constant. Let this constant $x_{G}$ be zero.

$x_{G}=x-M^{-1}\left[\left(m_{1} s_{1}+m_{2} l_{1}+m_{3} l_{1}\right) \sin \alpha_{1}+\left(m_{2} s_{2}+m_{3} l_{2}\right) \sin \alpha_{2}+m_{3} s_{3} \sin \alpha_{3}\right]=0$

In the flight phase, only one external force is applied to the biped - the gravity force. And this force is directed downwards. According to the law of conservation of momentum $[4,6]$, if at the beginning of the flight phase $x_{G}=0, \dot{x}_{G}=0$, then equality (6) will always be valid during this phase - "automatically".

By the differentiation of equalities (4), (5) and (6) we get the following relations:

$$
\dot{x}=0
$$

$$
\dot{y}=0
$$

and

$$
\begin{aligned}
\dot{x}_{G}= & \dot{x}-M^{-1}\left[\left(m_{1} s_{1}+m_{2} l_{1}+m_{3} l_{1}\right) \dot{\alpha}_{1} \cos \alpha_{1}+\right. \\
& \left.\left(m_{2} s_{2}+m_{3} l_{2}\right) \dot{\alpha}_{2} \cos \alpha_{2}+m_{3} s_{3} \dot{\alpha}_{3} \cos \alpha_{3}\right]=0
\end{aligned}
$$

By the differentiation of equalities (7), (8) and (9) we get:

$$
\ddot{x}=0
$$

$$
\ddot{y}=0
$$

and

$$
\begin{aligned}
\ddot{x}_{G}= & \ddot{x}-M^{-1}\left[\left(m_{1} s_{1}+m_{2} l_{1}+m_{3} l_{1}\right) \ddot{\alpha}_{1} \cos \alpha_{1}+\right. \\
& \left(m_{2} s_{2}+m_{3} l_{2}\right) \ddot{\alpha}_{2} \cos \alpha_{2}+m_{3} s_{3} \ddot{\alpha}_{3} \cos \alpha_{3}- \\
& \left(m_{1} s_{1}+m_{2} l_{1}+m_{3} l_{1}\right) \dot{\alpha}_{1}^{2} \sin \alpha_{1}- \\
& \left.\left(m_{2} s_{2}+m_{3} l_{2}\right) \dot{\alpha}_{2}^{2} \sin \alpha_{2}-m_{3} s_{3} \dot{\alpha}_{3}^{2} \sin \alpha_{3}\right]=0
\end{aligned}
$$

Equalities (5), (8) and (11) are valid only during support phase.

Introducing the row matrix

$$
\mathbf{J}(\alpha)=-M^{-1}\left[\begin{array}{lll}
\left(m_{1} s_{1}+m_{2} l_{1}+m_{3} l_{1}\right) \cos \alpha_{1} & \left(m_{2} s_{2}+m_{3} l_{2}\right) \cos \alpha_{2} & m_{3} s_{3} \cos \alpha_{3}
\end{array}\right],
$$

both equalities (9) and (12) can be rewritten in the following simple matrix form:

$$
\begin{gathered}
\dot{x}_{G}=\mathbf{J}(\alpha) \dot{\alpha}+\dot{x}=0 \\
\ddot{x}_{G}=\mathbf{J}(\alpha) \ddot{\alpha}+\dot{\mathbf{J}}(\alpha) \dot{\alpha}+\ddot{x}=0
\end{gathered}
$$


After the flight phase at the touchdown an absolutely inelastic impact is assumed. It means that the velocity $\dot{y}$ becomes zero after impact. At the instant of impact the velocity $\dot{y}$ changes stepwise. Also we assume that the leg tips do not slip. In our control strategy, the inter-link torque $\Gamma_{b}$ is finite and it does not have any influence on the result of the impact. The ground reaction $R\left(R_{x}, R_{y}\right)$ and torque $\Gamma_{c}$ at instant $t=T$ of an impact can be considered as an impulsive force and an impulsive torque respectively. These force and torque can be defined by the Dirac delta-functions $R_{x}=I_{R_{x}} \delta(t-T)$, $R_{y}=I_{R_{y}} \delta(t-T)$ and $\Gamma_{c}=I_{\Gamma_{c}} \delta(t-T)$. Here $\mathbf{I}_{R}\left(I_{R_{x}}, I_{R_{y}}\right)$ is the vector of magnitudes of the impulsive ground reaction in both leg tips, (see [14]), $I_{\Gamma_{c}}$ is the magnitude of an impulsive torque $\Gamma_{c}$. Values $I_{R_{x}}, I_{R_{y}}$ and $I_{\Gamma_{c}}$ are unknown quantities. The equations for the velocity jumps can be obtained through the integration of the matrix equations (3) and (12) (or (15)) over an infinitesimal time interval $\left(T^{+}-T^{-}\right)$. Consequently from the matrix equation (3) we get the algebraic equation in the following matrix form:

$$
\mathbf{D}(\alpha)\left(\dot{\mathbf{q}}^{+}-\dot{\mathbf{q}}^{-}\right)=\left[\begin{array}{r}
0 \\
1 \\
-1 \\
0 \\
0
\end{array}\right] I_{\Gamma_{c}}+\left[\begin{array}{ll}
0 & 0 \\
0 & 0 \\
0 & 0 \\
1 & 0 \\
0 & 1
\end{array}\right]\left[\begin{array}{l}
I_{R_{x}} \\
I_{R_{y}}
\end{array}\right]
$$

Here $\alpha$ is the configuration-vector of the biped at instant $T$ of the touchdown (impact). This configuration $\alpha$ does not change at the impact. Vectors $\dot{\mathbf{q}}^{-}$and $\dot{\mathbf{q}}^{+}$are the velocity vectors just before (at time $t=T^{-}$) and just after (at time $t=T^{+}$) the touchdown (impact) respectively. Just before the impact, the horizontal component of the velocity of point $a$ is $\dot{x}^{-}$, the horizontal component of the velocity of the center of mass $G$ is $\dot{x}_{G}^{-}$. These velocity components just after the impact are denoted by $\dot{x}^{+}$and $\dot{x}_{G}^{+}$ respectively. If the control strategy is such that $\dot{x}^{-}=\dot{x}^{+}$and $\dot{x}_{G}^{-}=\dot{x}_{G}^{+}$, then from equation (15) we obtain

$$
\dot{x}_{G}^{+}-\dot{x}_{G}^{-}=\mathbf{J}(\alpha)\left(\dot{\alpha}^{+}-\dot{\alpha}^{-}\right)+\dot{x}^{+}-\dot{x}^{-}=\mathbf{J}(\alpha)\left(\dot{\alpha}^{+}-\dot{\alpha}^{-}\right)=0
$$

Of course for the jumping of human the ground reaction is bounded. Therefore, our model of the touchdown can be considered as an asymptotical model.

\subsection{Control Strategy}

At time $t=0$, let the biped be in support phase and in vertical equilibrium position, i.e. (see Figure 3)

$$
\begin{aligned}
& x=0, y=0, \alpha_{1}=\alpha_{2}=\alpha_{3}=0 \\
& \dot{x}=0, \dot{y}=0, \dot{\alpha}_{1}=\dot{\alpha}_{2}=\dot{\alpha}_{3}=0
\end{aligned}
$$




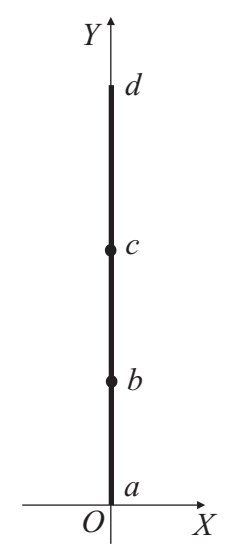

Fig. 3 Initial configuration of the biped.

At time $t>0$ the biped prepares a jump. It starts to crouch, using the feedback control

$$
\Gamma_{b}=-k_{p}\left(\varphi_{b}-\varphi_{b}^{d}\right)-k_{v} \dot{\varphi_{b}}
$$

Here $\varphi_{b}=\pi+\alpha_{1}-\alpha_{2}$ is the joint angle between links $a b$ and $b c, \varphi_{b}^{d}=$ const $<\pi$ is a desired angle $\varphi_{b}$ at the end of crouching, $k_{p}$ and $k_{v}$ are constant feedback gains. The constant value $\varphi_{b}^{d}$ is chosen adequately to bend the knees.

Equations (3), (10) and (11) are describing the process of the crouching of the biped. To satisfy identity $x_{G} \equiv 0$ we need to find the corresponding control torque $\Gamma_{c}$. So, the constraint (equality) (12) (or (15)) is added to equations (3), (10), (11). System (3), (10), (11), and (12) contains 8 scalar equations and 8 unknown variables: $\alpha_{1}, \alpha_{2}, \alpha_{3}, x$, $y, R_{x}, R_{y}$ and $\Gamma_{c}$. But it follows from equation (12) and by considering the dynamical balance of the motion of the center of mass $G$, that the horizontal component of the ground reaction $R_{x}=0$. Thus, we can omit equation (12) and integrate only equations (3), (10), (11) with $R_{x}=0$. If the center of mass is constantly located along the vertical line $x=0$ and the external torque in point $a$ is zero, then the angular momentum of the biped mechanism around point $a$ is equal to zero (according to the law of conservation of angular momentum [4], [6]). In this case, there is no rotation of the biped as a whole-body around the leg tips (around point $a$ ).

The feedback control (19) is applied until the instant when the term $\left(\varphi_{b}-\varphi_{b}^{d}\right)^{2}+\gamma \dot{\varphi}_{b}^{2}$ (the dimension of parameter $\gamma$ is $s^{2}$ ) becomes less than some previously given value $\Delta$. After this instant a thrust torque $\Gamma_{b}^{p}$

$$
\Gamma_{b}=\Gamma_{b}^{p}=\text { const }
$$

is applied (in open loop) in joint $b$. Identity $x_{G} \equiv 0$ is satisfied under the suitable control torque $\Gamma_{c}$. This control torque $\Gamma_{c}$ can be found using constraint (12). Note we do not prescribe torque $\Gamma_{c}$. It is calculated with constraint (12). The equations (3), (10), (11), (12) or (3), (10), (11) with $R_{x}=0$ can be integrated numerically to find the biped motion under control (20). Control (20) is applied until the instant when the vertical component $R_{y}$ of the ground reaction $R$ becomes zero. The flight phase starts after this instant. 
In the flight phase, matrix equation (1) of the motion is valid. Equality (6) and consequently (12) are valid in this phase as noted above. At the instant of the start of the flight phase the angular momentum around point $a$ is zero. During all the flight phase without any external torque this angular momentum will be zero. Then the angular momentum around the center of mass $G$ is also equal to zero because $x_{G}=0$. Due to this, during the flight phase the biped does not rotate as a whole around its center of mass.

During the flight phase, the feedback control (19) with $\varphi_{b}^{d}=\pi$ is used to straighten up the biped. Constraint (10) is added to matrix equation (1) to allow the biped to obtain the same position in frame $X O Y$ after touchdown and to straighten up (in the next support phase). System (1), (10) contains six scalar equations with six unknown variables: $\alpha_{1}, \alpha_{2}, \alpha_{3}, x, y$ and $\Gamma_{c}$.

Remark: When a human jumps in order to touch the ceiling with one hand, in the flight phase, he straightens up his legs and lets his other arm in a low position along his torso. It is possible to explain this in the following way. According to the law of variation of momentum ( $[4],[6]$ ), the motion of the center of mass of the biped in the flight phase does not depend on the relative motions of its links (if the air resistance is negligible). Thus, if the legs straighten up and one arm moves down, then another arm raises up higher.

At a certain instant of the flight phase, altitude $y_{G}$ of the center of mass becomes maximal. After this instant, the altitude of the center of mass decreases and the biped prepares a touchdown. To prepare a touchdown feedback control (19) with an adequate value $\varphi_{b}^{d}=$ const $<\pi$ is used. When the leg tips (point $a$ ) touches the ground (at the origin $x=0, y=0$ ) an absolutely inelastic impact occurs. This impact is described by algebraic equations (16) and (17). The five components of vector $\dot{\mathbf{q}}^{-}$are the components of the velocity-vector at the end of flight phase. We get these quantities from the numerical solution of equations (1), (10) (note $\dot{x}^{-}=\dot{x}_{G}^{-}=0$ ). It is necessary to find the components of vector $\dot{q}^{+}$. But, according to the assumption about the absolutely inelastic impact, $\dot{y}^{+}=0$. Also we want to obtain after an impact $\dot{x}^{+}=\dot{x}_{G}^{+}=0$. Then there are only three unknown components: $\dot{\alpha}_{1}^{+}, \dot{\alpha}_{2}^{+}, \dot{\alpha}_{3}^{+}$in this vector $\dot{\mathbf{q}}^{+}$. System (16), (17) contains 6 scalar equations with 6 unknown variables: $\dot{\alpha}_{1}^{+}, \dot{\alpha}_{2}^{+}, \dot{\alpha}_{3}^{+}, I_{R_{x}}, I_{R_{y}}$ and $I_{\Gamma_{c}}$. However, if $\dot{x}_{G}^{+}=0$, then $I_{R_{x}}=0$. Thus, we can omit equation (17) and consider only equations (16) with equality $I_{R_{x}}=0$.

The next support phase starts after touchdown. During this support phase the goal of the control is to straighten up the biped, that is to bring it to the initial configuration

$$
\alpha_{1}=\alpha_{2}=\alpha_{3}=0
$$

To do this it is possible to use the feedback control (19) with $\varphi_{b}^{d}=\pi$. Equations (3), (10), (11) with $R_{x}=0$ can be used to find motion of the biped during this support phase. Torque $\Gamma_{c}$ can be also calculated from these equations.

Thus, the structure of the control torques $\Gamma_{b}$ and $\Gamma_{c}$ in the knees and hips changes from one phase to another. The control torque $\Gamma_{b}$ has the form of the linear feedback (see (19)) in the crouching, the flight, and after the touchdown, but with different presets $\varphi_{b}^{d}$ for different phases. Besides, during the crouching, we limit this control torque to prevent the canceling of the ground reaction and unplanned takeoff of the biped. After the crouching, the torque in the knees varies quickly: the thrust torque (20) in open loop is applied in joint $b$. We calculate the torque $\Gamma_{c}$ in order to maintain 
a null horizontal component of the ground reaction in support phase and at the instant of the touchdown. In the flight phase we calculate the control torque $\Gamma_{c}$ to ensure equality $x=0$.

\subsection{Energy Recovery Using Springs in Joints}

The problem of recovery of the energy is discussed in several papers such as [28], [39], [38], [29], [37], [13] or [34]. Here we study the problem of the energy recovery, using passive springs in joints $b$ and $c$.

Using Hooke's law, the expressions of the torques developed by these linear springs are

$$
\Gamma_{b}^{s}=k_{b}^{s}\left(\pi-\varphi_{b}\right), \Gamma_{c}^{s}=k_{c}^{s}\left(\pi-\varphi_{c}\right)
$$

where $k_{b}^{s}=$ const $>0$ and $k_{c}^{s}=$ const $>0$ are the stiffness coefficients of the springs in joints $b$ and $c$ respectively. For the two intervals

$$
0 \leq k_{b}^{s} \leq k_{\text {bmax }}^{s}, \quad 0 \leq k_{c}^{s} \leq k_{c \max }^{s}
$$

with $k_{b \max }^{s}=k_{c \max }^{s}=100 \mathrm{~N} \cdot \mathrm{m} / \mathrm{rad}$ we calculated the energy consumed in joints $b$ and $c$ by torques $\Gamma_{b}$ (see the controls (19) and (20)) and $\Gamma_{c}$ during the jumping process.

\subsection{Physical Parameters of the Biped}

The parameters of the biped, used in simulation are supplied from the book [14]. Table 1 gathers these physical data, which are valid for a normal adult male (the gravity acceleration is $9.81 \mathrm{~m} \cdot \mathrm{s}^{-2}$ ). For the lower leg the mass of the foot is included in $m_{1}$.

\begin{tabular}{|c||c|c|c|}
\hline & $\begin{array}{c}\text { Link } a b \\
\text { (coupled shins) }\end{array}$ & $\begin{array}{c}\text { Link } c b \\
\text { (coupled thighs) }\end{array}$ & $\begin{array}{c}\text { Link } c d \\
\text { (trunk) }\end{array}$ \\
\hline Length $(\mathrm{m})$ & $l_{1}=0.5$ & $l_{2}=0.41$ & $l_{3}=0.84$ \\
\hline Mass $(\mathrm{kg})$ & $m_{1}=9.2$ & $m_{2}=17.2$ & $m_{3}=48.6$ \\
\hline $\begin{array}{c}\text { Inertia moment } \\
\text { defined about } \\
\text { the center of mass } \\
\left(\mathrm{kg} \cdot \mathrm{m}^{2}\right)\end{array}$ & $I_{1}=1.1$ & $I_{2}=0.51$ & $I_{3}=3.91$ \\
\hline $\begin{array}{c}\text { Center of mass } \\
\text { location }(\mathrm{m})\end{array}$ & $s_{1}=0.18$ & $s_{2}=0.23$ & $s_{3}=0.39$ \\
\hline
\end{tabular}

Table 1 Biped's parameters 
3 Results

We show here the results of the simulation with the following parameters of control: angle $\varphi_{b}^{d}$ for crouching is equal to $2.36 \mathrm{rad}$, angle $\varphi_{b}^{d}$ for the preparation of the touchdown equals $2.53 \mathrm{rad}$ (both these values are taken from [25]), $k_{p}=40000 \mathrm{~N} \cdot \mathrm{m} / \mathrm{rad}$, $k_{v}=4000 \mathrm{~N} \cdot \mathrm{m} \cdot \mathrm{s} / \mathrm{rad}, \Delta=5^{-4}$, in open loop $(20) \Gamma_{b}^{p}=300 \mathrm{~N} \cdot \mathrm{m}$,

The stick diagram in Figure 4 is derived from the simulation of the biped jump (without springs in the joints) and depicts several biped's configurations during the jump. This diagram seems natural for the human jump. The legs bend the knee forward $\left(\varphi_{b}=\pi+\alpha_{1}-\alpha_{2} \leq \pi\right)$ all the time, angle $\varphi_{c}$ in the hip joints is such as $\varphi_{c}=$ $\pi+\alpha_{2}-\alpha_{3} \geq \pi$. (Note that these "human's features" of the jump are not prescribed previously by the statement of the problem.) 


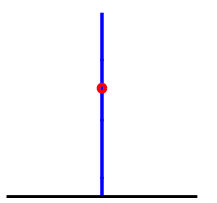

a) initial configuration

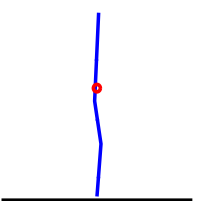

c) flight phase after the thrust

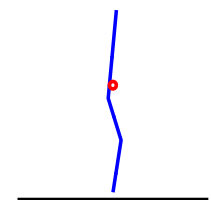

e) flight phase, preparation of the touchdown

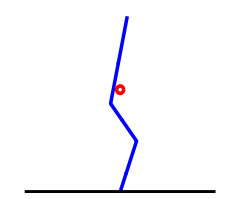

b) end of crouching

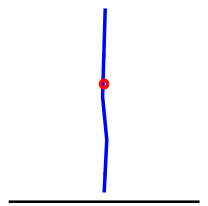

d) flight phase, almost on straight line configuration

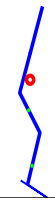

f) touchdown

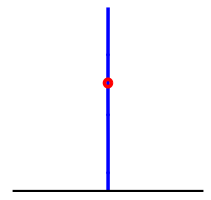

g) final configuration coincides with the initial configuration

Fig. 4 Jumping motion as a sequence of stick figures; the position of the center of mass is depicted with a dot. 
On Figures, 5-10, which are associated to the upward jump, there are three vertical dashed lines to highlight successively the crouching phase, the thrust phase, the flight phase, the touchdown and the straightening up movement. The rise of the center of mass of the biped (the difference between the current and initial altitude) and the rise of the leg tips are presented in Figure 5. The maximal rise of the center of mass is equal to $0.0913 \mathrm{~m}$. During the flight phase, the rise is similar for the center of mass and for the leg tips. Before the thrust with control (20) the altitude of the center of mass becomes less than at the initial configuration. Just after the touchdown this altitude also becomes less than at the initial and final configurations. As noted above, the motion of the center of mass $G$ of the biped in the flight phase does not depend on the relative motion of the links (if air resistance is neglected). However, duration of the flight phase of course depends on the positions of the links. The nearer the leg tips (point $a$ ) to the ground before the touchdown, the shorter the duration of the flight phase.

The velocity of the center of mass as functions of time is shown in Figure 6 .

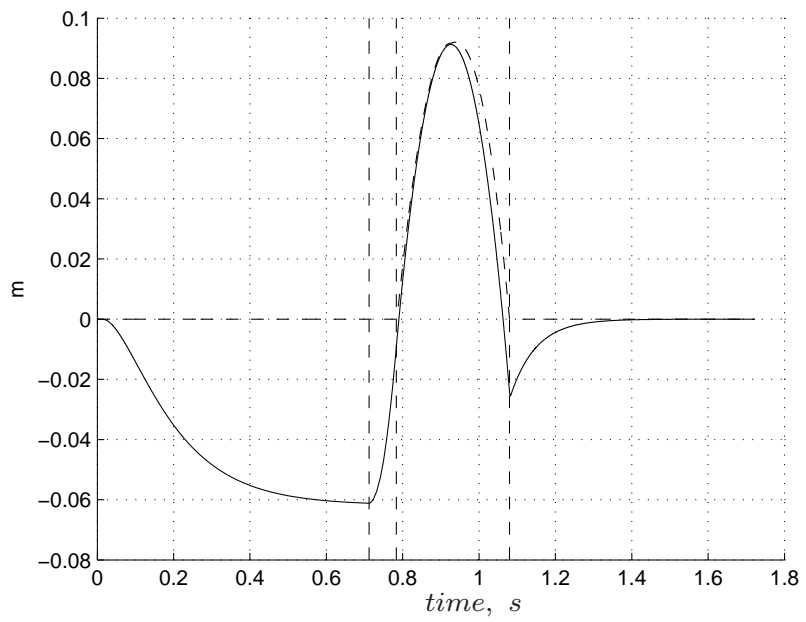

Fig. 5 Rise of the center of mass (solid line), of the leg tips (dashed line) during jump. 


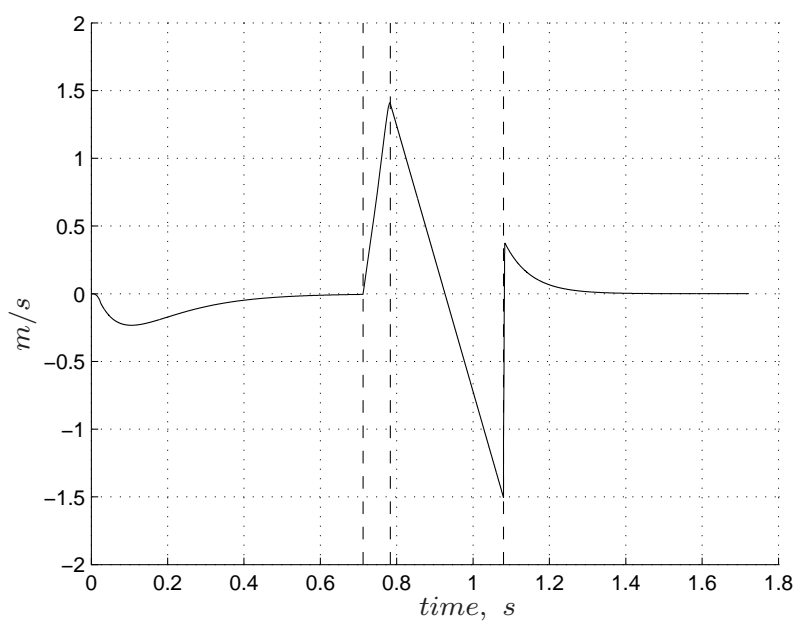

Fig. 6 Velocity of the center of mass during jump.

Figure 7 shows the vertical component $R_{y}$ of the ground reaction $R$. Naturally, the shape of this graph is the same as the shape of the graph of the acceleration of the center of mass. The graph of the ground reaction $R_{y}$ for the jumping motion is logical physically because $R_{y}>0$ in stance phase and $R_{y}=0$ in flight phase. At the initial time this component is equal to the weight of the biped mechanism, which is $735.75 \mathrm{~N}$. At the beginning of the crouching it decreases, which is reasonable because the velocity of the center of mass at this time decreases (see Figure 6) and consequently the acceleration of the center of mass at this time is negative. The minimum value of the component $R_{y}$ is close to zero. Therefore, we assign a minimum value to feedback control (19) at this time to avoid the canceling of the ground reaction and the corresponding takeoff of the biped. At the end of the phase of crouching the ground reaction becomes equal (approximately) to the biped's weight. The ground reaction becomes large during the thrust control $(20)$, which begins at around $0.71 \mathrm{~s}$. Then at time instant $0.783 \mathrm{~s}$ it becomes zero. During the flight phase the ground reaction is naturally zero. At the instant of the touchdown (at around $1.08 \mathrm{~s}$ ), the vertical component of the ground reaction and the acceleration of the center of mass equal $+\infty$. At the end of the process the ground reaction becomes again equal to the weight of the mechanism.

The profile of angle $\varphi_{b}$ in the knee joints (see Figure 8) allows us to show clearly the phases of crouching, of the thrust with control (20), of the flight phase, of the touchdown and the straightening up movement of the biped. During flight phase, the straight line configuration such as $\varphi_{d}=\pi$ is not exactly reached. Just after the time when the vertical component of the velocity of the center of mass $\dot{y}_{G}$ becomes negative, the control law starts to prepare the touchdown. At this time angle $\varphi_{b}$ in the knees decreases. After the touchdown the value of $\varphi_{b}$ tends to $\pi$.

The profiles of the torques $\Gamma_{b}$ and $\Gamma_{c}$ are shown in Figure 9. We can see the torques during the crouching, the preparation of the flight phase by applying in open loop control torque $\Gamma_{b}=\Gamma_{b}^{p}=300 N \cdot m$, the flight phase, the touchdown and the straightening up movement of the biped. The magnitude of torque $\Gamma_{c}$ at the instant 


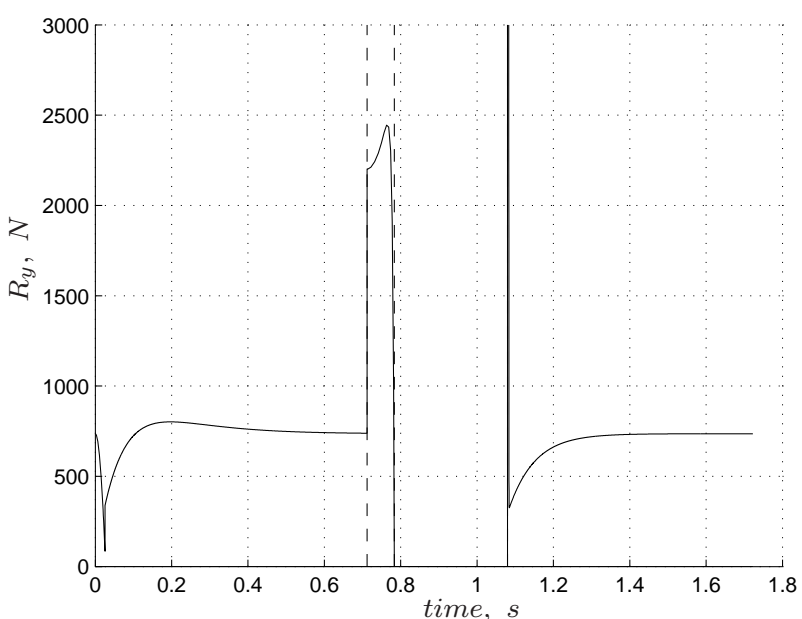

Fig. 7 Vertical component $R_{y}$ of the ground reaction $R$.

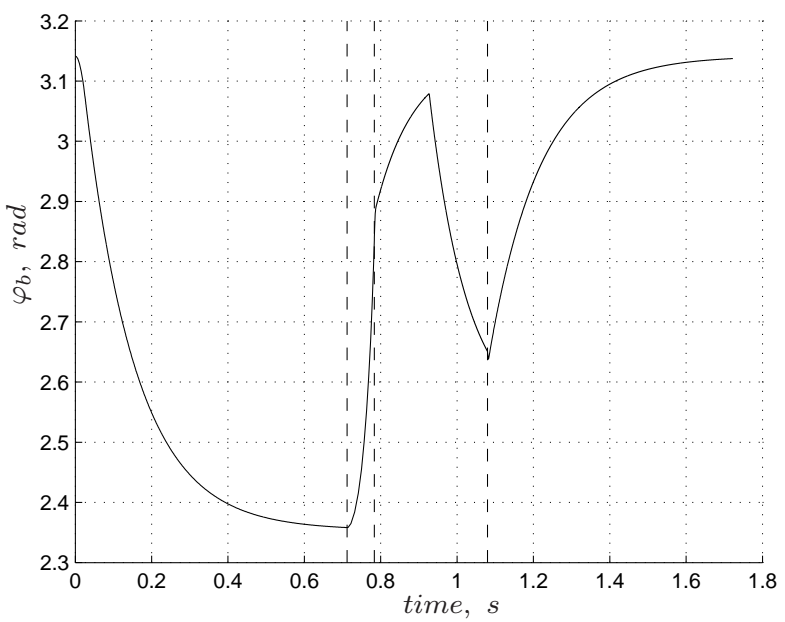

Fig. 8 Profile of the joint angle $\varphi_{b}$.

of the touchdown becomes infinite. Assuming that there is no recuperation of the mechanical energy when the actuating torques are " break-like ", the energy used to generate and control the movement is the integral of the absolute joint powers on the time cycle, see [14] and [9]

$$
W(t)=\int_{0}^{t}\left[\left|\Gamma_{c} \dot{\varphi}_{c}\right|+\left|\Gamma_{b} \dot{\varphi}_{b}\right|\right] d \tau
$$



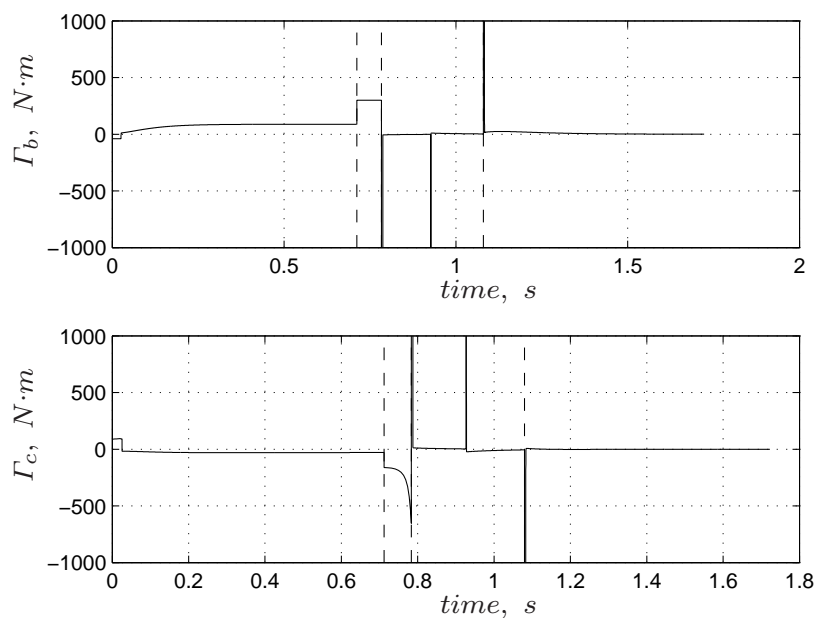

Fig. 9 Profiles of the joint torques $\Gamma_{b}$ and $\Gamma_{c}$.

where $\varphi_{c}=\pi+\alpha_{2}-\alpha_{3}$ and $\dot{\varphi}_{c}=\dot{\alpha}_{2}-\dot{\alpha}_{3}$. At the instant of the touchdown (impact) $t=T$, torque $\Gamma_{c}=I_{\Gamma_{c}} \delta(t-T)$. To calculate the following energy

$$
W(T)=\int_{T^{-}}^{T^{+}}\left|\Gamma_{c} \dot{\varphi}_{c}\right| d t
$$

consumed at the impact we use the formulas [14]:

$$
\begin{gathered}
W(T)=\left|I_{\Gamma_{c}} \frac{\dot{\varphi}_{c}\left(T^{-}\right)+\dot{\varphi}_{c}\left(T^{+}\right)}{2}\right|, \text { if } \dot{\varphi}_{c}\left(T^{-}\right) \dot{\varphi}_{c}\left(T^{+}\right) \geq 0 \\
W(T)=\left|I_{\Gamma_{c}} \frac{\dot{\varphi}_{c}^{2}\left(T^{-}\right)+\dot{\varphi}_{c}^{2}\left(T^{+}\right)}{2\left[\dot{\varphi}_{c}\left(T^{-}\right)-\dot{\varphi}_{c}\left(T^{+}\right)\right]}\right|, \text {if } \dot{\varphi}_{c}\left(T^{-}\right) \dot{\varphi}_{c}\left(T^{+}\right)<0
\end{gathered}
$$

Figure 10 depicts the energy consumption of the knee and hip actuators, which is defined by (24) and from the instant of impact with the additional quantity (26), during the jumping process as a function of time. 


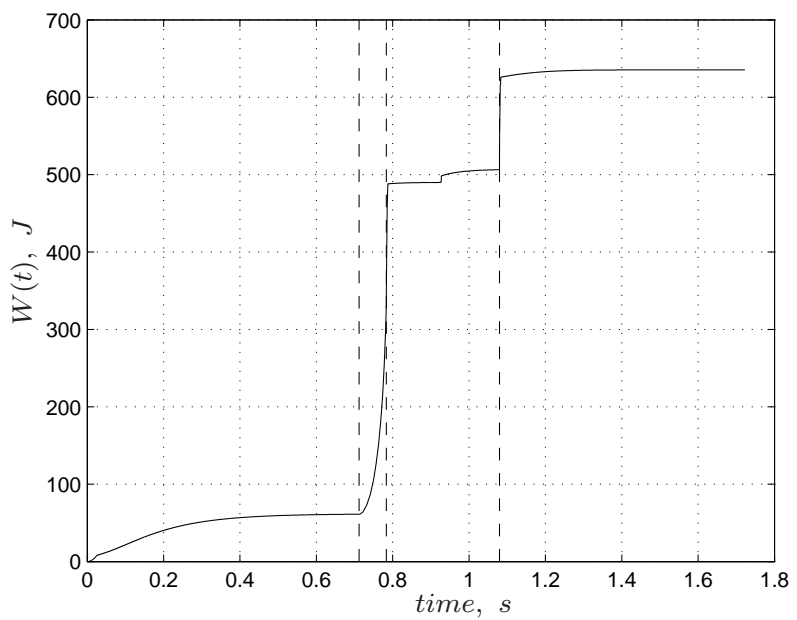

Fig. 10 Energy consumption in the knee and hip joints.

Function $W(t)$ is naturally strictly monotonous. The most part of the energy is consumed at the time of the thrust control (20). At the instant of the touchdown this function changes stepwise. But the energy consumed at the instant of the touchdown is much less than the energy consumed at the time of the thrust. During crouching and after the touchdown, the energy consumption is relatively small. The final value of $W(t)$ is equal to $635.5 \mathrm{~J}$.

The duration of the trust phase decreases when the trust torque increases. Then if the thrust torque $\Gamma_{b}^{p}$ in control (20) increases, the operating time $\Delta t$ of this control decreases strictly monotonically. For example, in the case $\Gamma_{b}^{p}=300 \mathrm{~N} \cdot \mathrm{m}$, the operating time $\Delta t=0.064 \mathrm{~s}$, in the case $\Gamma_{b}^{p}=600 \mathrm{~N} \cdot \mathrm{m}$, the operating time $\Delta t=0.043 \mathrm{~s}$.

In Figure 11, we show the energy consumed in the hip and knee joints as a function of the thrust torque $\Gamma_{b}^{p}$. This energy increases, if the torque $\Gamma_{b}^{p}$ increases. The dependency shown in Figure 11 is approximately linear. 


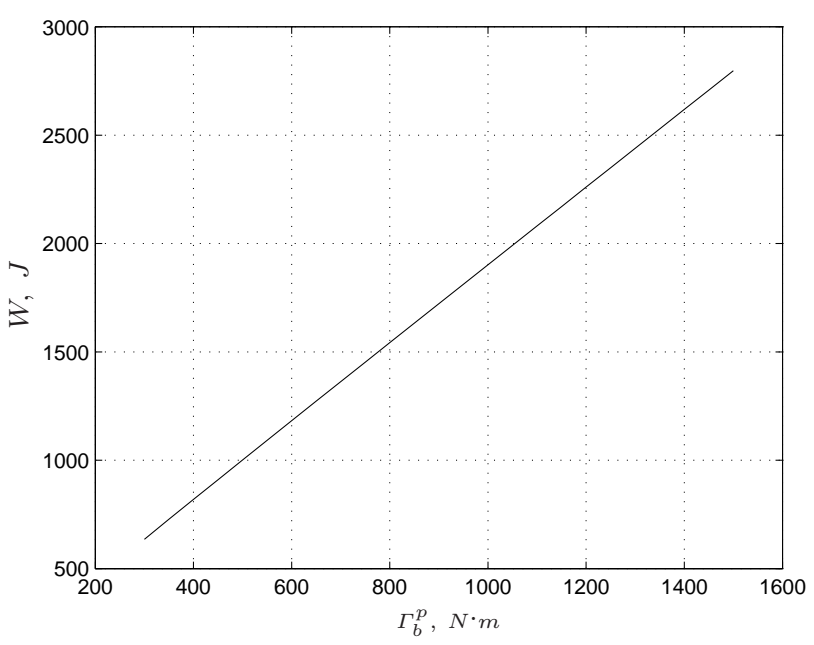

Fig. 11 Energy consumed in the hip and knee joints as a function of $\Gamma_{b}^{p}$.

In Figures 12 and 13 we show several results obtained for the case when the spring in the joints are used.

For the five values $k_{b}^{s}=0,25 \mathrm{~N} \cdot \mathrm{m}, 50 \mathrm{~N} \cdot \mathrm{m}, 75 \mathrm{~N} \cdot \mathrm{m}$ and $100 \mathrm{~N} \cdot \mathrm{m}$, Figure 12 shows the mechanical energy $W$ consumed in the knee and hip joints during the jump as functions of gain $k_{c}^{s}$, with $\Gamma_{b}=\Gamma_{b}^{p}=300 N \cdot m$ (in open loop (20)).

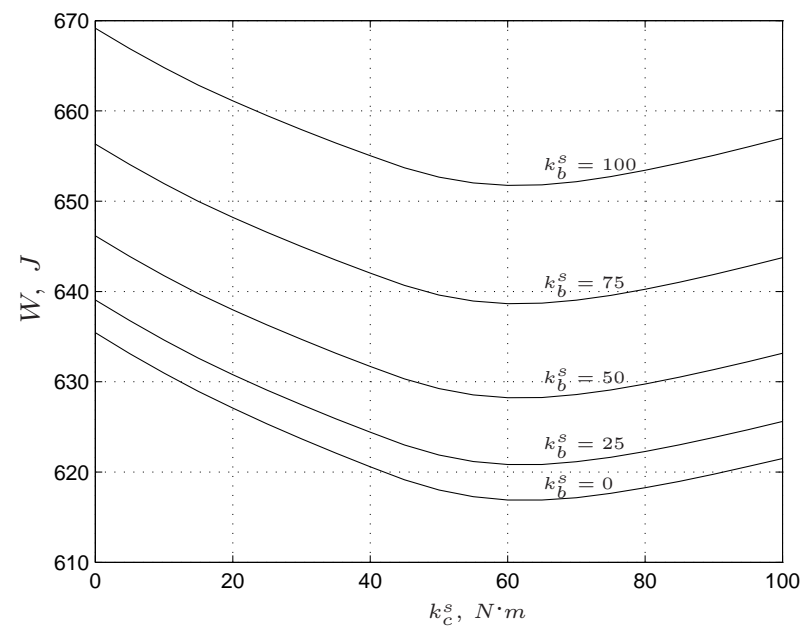

Fig. 12 Mechanical energy consumed in the knee and hip joints during the jumping process as functions of gain $k_{c}^{s}$ for the five values of $k_{b}^{s}$. 
We can observe that, for each value $k_{b}^{s}$ there is an optimal value for $k_{c}^{s}$ that minimizes the consumed mechanical energy. But the economy of energy is close to $3 \%$ only. We also see from Figure 12 that for all values of $k_{b}^{s}$ this optimal value $k_{c}^{s} \approx 61 \mathrm{~N} \cdot \mathrm{m}$. Figure 12 also shows that the greater the gain $k_{b}^{s}$, the more important the gap of energy.

Figure 13 shows the maximum rise of the center of mass depending on coefficient $k_{b}^{s}$ with $\Gamma_{b}^{p}=300 N \cdot m$ and $k_{c}^{s}=61 N \cdot m$.

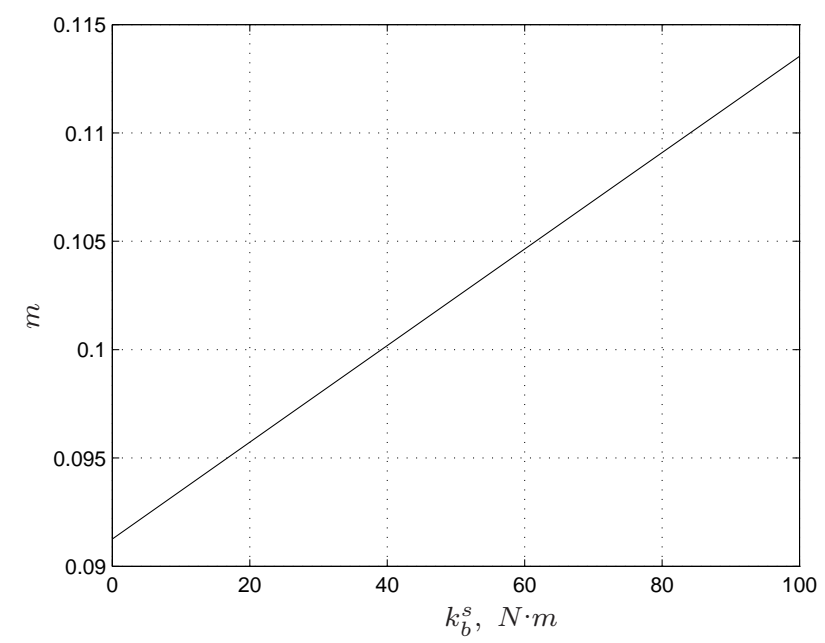

Fig. 13 Maximum rise of the center of mass of the bipedal mechanism during the jump as a function of gain $k_{b}^{s}$ with $k_{c}^{s}=61 N \cdot m$.

We observe on Figure 13 that if gain $k_{b}^{s}$ increases, the maximum rise of the center of mass also increases, and approximately linearly.

\section{Discussion}

In order to check the validity of the simulation results, they are now compared with respect to experimental data. Linthorne [23] determines with a force platform the profile of the ground reaction force during the counter movement phase labeled from $a$ to $c$, the jumping phase between $c$ and $d$ and the takeoff impulse phase around point $d$ for the maximal capacities of the human vertical jump, see Figure 15. 


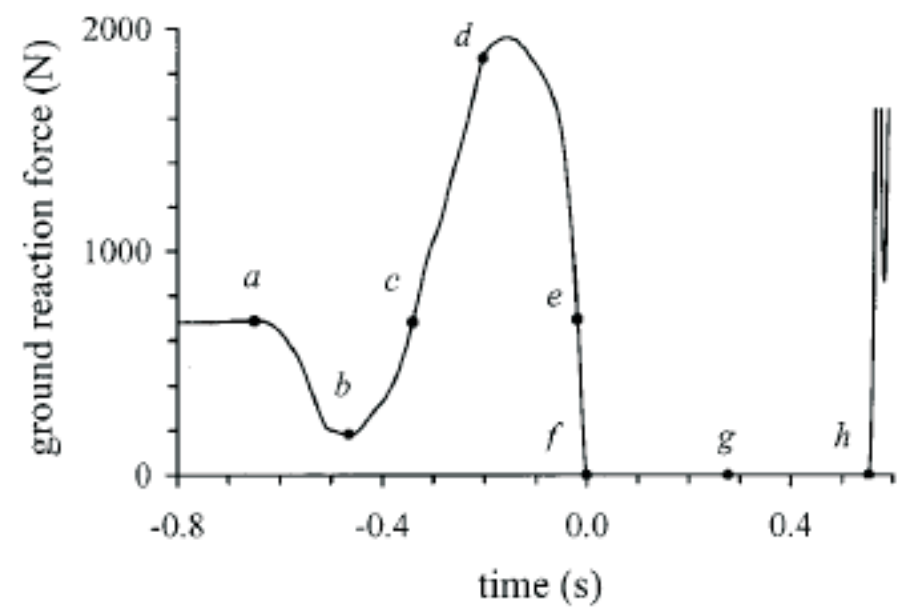

Fig. 14 Ground reaction force for a human maximal capacities vertical jump obtained using a force platform, see Linthorne [23].

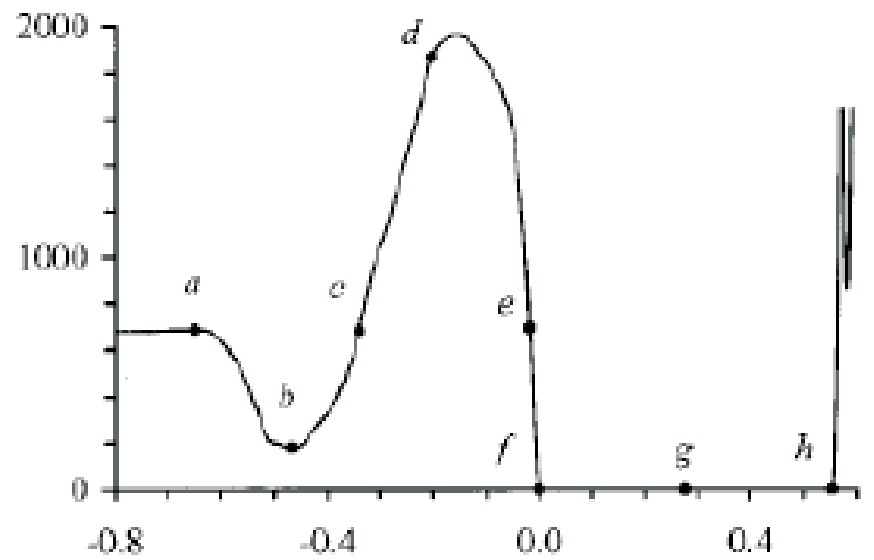

Fig. 15 Ground reaction force for a human maximal capacities vertical jump obtained using a force platform, see Linthorne [23].

The total duration of these three phases is close to $0.8 s$ as in our simulation (see Figure 7). The peak vertical ground force measured just at the beginning of the takeoff impulse phase is equal to $2000 \mathrm{~N}$. In our modeling this peak is equal to $2445 \mathrm{~N}$ approximately. The shape of the profile of the ground reaction force in Figure 15 is not far from the shape of the profile in Figure 7. Perhaps, the difference between the shapes of these profiles is due to absence in our model of the arms, the feet and the muscles [33]. Furthermore another source of discrepancy between Figure 15 and Figure 7 could be 
the use of different inertia moments, masses, lengths and localization of the centers of mass.

In [2], authors proposed data about the experimental results for the vertical jump of five human subjects. Measures of surface electrodes of electromyographic (EMG) signals, force-plate, and kinematic data were recorded simultaneously during each jump. From the obtained data, the authors observed the following results. Just before lift-off the peak vertical ground forces measured for subjects range from 1500 to $2100 \mathrm{~N}$. Figure 7 shows that for our model with $\Gamma_{b}^{p}=300 N \cdot m$, the peak of the vertical component $R_{y}$ equals $2445 \mathrm{~N}$, which is not far from these values. The peak fore-aft components measured for the subjects range from 120 to $270 \mathrm{~N}$. With the statement of our problem, the horizontal component $R_{x}$ is null.

The vertical velocities of the whole-body center of mass at lift-off belongs to the range from 2.0 to $2.5 \mathrm{~m} / \mathrm{s}$. The peak vertical accelerations at lift-off of the wholebody center of mass, measured for the subjects range from 15 to $19 \mathrm{~m} / \mathrm{s}^{2}$. A peak vertical velocity in our simulation with $\Gamma_{b}^{p}=300 \mathrm{~N} \cdot \mathrm{m}$ at lift-off is almost $1.4 \mathrm{~m} / \mathrm{s}$. The corresponding acceleration at lift-off is equal to $23 \mathrm{~m} / \mathrm{s}^{2}$ approximately. Thus, the order of magnitude of the peak velocities and accelerations for our model is the same as for the experiments. The mean of the jump height for the subjects is $37 \mathrm{~cm}$. With our model for $\Gamma_{b}^{p}=300 \mathrm{~N} \cdot \mathrm{m}$ this jump height is $10 \mathrm{~cm}$ approximately. For $\Gamma_{b}^{p}=600 \mathrm{~N} \cdot \mathrm{m}$, this jump height is $22 \mathrm{~cm}$.

The given above comparison shows satisfactory agreements between our simulation results and the experimental data from the human jump. Thus, our model of the biped jump gives coherent results for vertical jumping.

Is it efficient to add springs to joints of a bipedal robot to jump? It is shown in Section 3 that without springs $\left(k_{c}^{s}=0, k_{b}^{s}=0\right)$ and with the control torque $\Gamma_{b}^{p}=$ $300 N \cdot m$ (see an open loop (20)) the maximum rise of the center of mass equals $0.0913 \mathrm{~m}$ and the corresponding energy consumed by the drives in joints $b$ and $c$ equals $635.5 \mathrm{~J}$. Using the springs located in the hip and knee joints with the stiffness coefficients $k_{c}^{s}=61 \mathrm{~N} \cdot \mathrm{m} / \mathrm{rad}$ and $k_{b}^{s}=75 \mathrm{~N} \cdot \mathrm{m} / \mathrm{rad}$ we can get the same maximum rise of $0.0913 \mathrm{~m}$ of the center of mass, but with a smaller control torque $\Gamma_{b}^{p}=260 \mathrm{~N} \cdot \mathrm{m}$. The corresponding energy consumed in the joints is also less and almost equal to $564 \mathrm{~J}$. So, the economy of mechanical energy is almost $11.4 \%$ to get the same maximum rise.

Their influence is greater to improve the performances of jumping than to recover energy.

\section{Conclusions}

\section{- A. Conclusions}

This paper deals with the vertical upward jump of a planar biped. All the phases to describe the jumping process are defined. The control torques are applied in the hip and knee joints of the studied mechanism. The mechanical model of the biped jump is chosen to be relatively simple and at the same time relatively close to human in order to evaluate the important phenomena in the upward jump. The dynamic model for this kind of jumping bipeds is developed through a compact matrix form. The control strategy is designed to organize the jump. The designed control law keeps the center of mass of the biped always on the same vertical axis. The stick diagram, based on simulations results, seems natural for the human upward jump. Our model shows that the major part of energy is consumed at the times 
of the thrust and the touchdown. The energy consumption at the instant of the touchdown is much less than the energy consumption at the time of the thrust. We have compared several characteristics of the jumping of our three-link biped with experimental characteristics of the jumping of human. There is coherence between these characteristics.

The problem of the energy recovery is considered for the jumping of the biped through the addition of springs at the hip and the knee joints. Their contribution can be viewed as a portion of the driving torques. The springs do not have a large influence to minimize the mechanical energy consumed during the jump. The springs at the knees help to increase the rise of the bipedal mechanism.

- B. Future Works

In this paper we have considered the biped model without feet. Thus the missing of the controlled feet is a drawback of our study. But in the near future we intend to extend the results to a more complex model with controlled feet. Feet-rotation phases will be considered. Also we plan to study the forward jump.

\section{References}

1. Ali, B. E. Contribution à la Commande du Centre de Masse d'un Robot Bipède. PhD thesis, Thèse de doctorat, Institut National Polytechnique de Grenoble, 1999.

2. Anderson, F. C., And PAndy, M. G. A dynamic optimization solution for vertical jumping in three dimensions. Computer Methods in Biomechanics and Biomedical Engineering 2 (2009), 201-231.

3. Aoustin, Y., Chevallereau, C., and Formal'skit, A. M. Numerical and experimental study of the virtual quadruped - semiquad. Multibody System Dynamics 16 (2006), 1-20.

4. Appell, P. Dynamique des Systèmes - Mécanique Analytique. Paris, P. Gauthiers-Villars, 1931.

5. Boone, G. N., And Hodgins, J. K. Slipping and tripping reflexes for bipedal running robots. J. of Autonomous Systems 4, 3 (1997), 51-66.

6. Chetaev, N. G. Theoretical Mechanics. Springer Verlag (Mir Plublisher), 1989.

7. Chevallereau, C., Abba, G., Aoustin, Y., Plestan, F., Westervelt, E., CanuddasDE Wit, C., AND GRIzZLE, J. Rabbit: a testbed for advanced control theory. IEEE Control Systems Magazine 23, 5 (2003), 57-79.

8. Chevallereau, C., And Aoustin, Y. Optimal reference trajectories for walking and running of a biped. Robotica 19, 5 (2001), 557-569.

9. Chevallereau, C., Bessonnet, G., Abba, G., And Aoustin, Y. Bipedal robots: modeling, design and building walking robots. ISTE and Wiley Editions, New-York, 2009.

10. Collins, S. H., Wisse, M., And Ruina, A. A three dimensional passive-dynamic walking robot with two legs and knees. Int. J. of Robotics Research 20, 7 (2001), 607-615.

11. Demura, K., Tachi, N., Maekawa, T., and Ueno, T. Kensi-chan: Design of a humanoid for running. RoboCup 2001: Robot Soccer World World Cup V, Lecture Notes in Control and Information Sciences 2377 (2006), 283-315.

12. Engell-Norregard, M., Niebe, S., and Erleben, K. A joint-constraint model for human joints using signed distance-fields. Multibody System Dynamics, DOI 10.1007/s11044-0119296-1 (2011).

13. Farrel, K. D., Chevallereau, C., and Westervelt, E. R. Energetic effects of adding springs at the passive ankles of a walking biped robot. In Proc. of the IEEE Conf. on Robotics and Automation (Roma, Italy, 2007), pp. 3591-3596.

14. Formal'skiI, A. Locomotion of Anthropomorphic Mechanisms. Nauka, Moscow, 1982, (In Russian).

15. Goswami, D., and Vadakkepat, P. Planar bipedal jumping gaits with stable landing. IEEE Trans. on Robotics 25, 5 (2009), 1030-1046.

16. Grishin, A., Formal'skit, A., Lensky, A., And Zhitomirsky, S. Dynamic walking of a vehicle with two telescopic legs controlled by two drives. Int. J. of Robotics Research 13, 2 (1994), 137-147. 
17. Hurst, J. W., Chestnutt, J. J., And Rizzi, A. A. Design and philosophy of the bimasc, a higly dynamic biped. In Proc. of the IEEE Conf. on Robotics and Automation (Roma, Italy, 2007), pp. 1863-1868.

18. Itiki, C., Kalaba, R., And Udwadia, F. Inequality constraints in the process of jumping. Applied Mathematics and Computation 78 (1996), 163-173.

19. Jackson, M., Benkhemis, I., Begon, M., Sardain, P., Vallée, C., and Lacouture, P. Identifying the criterion spontaneously minimized during the take-off phase of a sub-maximal long jump through optimal synthesis. Multibody System Dynamics, DOI 10.1007/s11044-011-9278-3 (2011).

20. Kajita, S., Nagasaki, T., Kaneko, K., Yokio, K., and Tanie, K. A hop towards running humanoid biped. In Proc. of the IEEE Conf. on Robotics and Automation (2004), pp. 629635.

21. Ker, R., Bennett, M. B., Bibby, S. R., Kerster, R. C., And Alexander, R. M. The spring in the arc of the human foot. Nature, 325 (1987), 147-149.

22. Khang, G., And Zajac, F. E. Paraplegic standing controlled by functional neuromuscular stimulation: Part 1 - computer model and control-sytem design. IEEE Trans. on Biomedical Engineering 36, 9 (1989), 873-884.

23. Linthorne, N. P. Analysis of standing vertical jumps using a force platform. Am. J. Phys 69, 11 (2001), 1198-1204.

24. McGeer, T. Passive dynamic walking. Int. J. of Robotics Research 9, 2 (1990), 62-82.

25. Meghdari, A., And Aryanpour, M. Dynamic modeling and analysis of the human jumping process. J. of Intelligent and Robotics Systems 37 (2003), 97-115.

26. Nagasaka, K., Kuroki, Y., Suzuki, S., Itoh, Y., and Yamaguchi, J. Integrated motion control for walking, jumping and running on small bipedal entertainment robot. In Proc. of the IEEE Int. Conf. on Robotics and Automation (2004), pp. 3189-3194.

27. Pandy, M. G., And Zajac, F. E. Optimal muscular coordination strategies for jumping. J. Biomechanics 24, 1 (1991), 1-10.

28. Pratt, G., and Williamson, M. Series elastic actuators. In Proc. IEEE Conf. on Intelligent Robots and Systems (1995), vol. 1, pp. 399-406.

29. Pratt, J. E., and Krupp, B. T. Series elastic actuators for legged robots. In Proc. of SPIE Volume. (2004), vol. 5422, pp. 135-144.

30. Raibert, M. H., Brown, J. H. B., and Chepponis, M. Experiments in balance with a 3d one-legged hopping machine. Int. J. of Robotics Research 3, 2 (1984), 75-92.

31. Rogozhin, D. D. Impulsive control for anthropomorphic mechanism in the running with instantaneous support phase. Theory and Practice in Physical Training (In Russian), 5 (1989), 27-38.

32. Sabourin, C., Bruneau, O., and Buche, G. Control strategy for the robust dynamic walk of a biped robot. Int. J. of Robotics Research 25, 9 (2006), 843-860.

33. SAKKA, S., AND YoKor, K. Humanoid vertical jumping based on force feedback and inertial forces optimization. In Proc. of the IEEE Int. Conf. on Robotics and Automation (Barcelona, 2005), pp. 3752-3757.

34. SchauB, T., Scheint, M., Sobotka, M., Seiberl, W., and Buss, M. Effects of compliant ankles on bipedal locomotion. In Proc. of the IEEE Int. Conf. on Robotics and Automation (2009), pp. 2761-2766.

35. Song, S., And Waldron, K. Machines That Walk: The Adaptive Suspension Vehicle. MIT Press Cambridge, MA, USA, 327 pages, 1988.

36. VAn der Linde, R. Q., Wisse, M., And Schwab, A. L. A 3D passive dynamic biped with yaw and roll compensation. Robotica 19 (2001), 275-284.

37. Vanderborght, B. Dynamic stabilization of the biped Lucy powered by actuators with controllable stiffness. PhD thesis, Vrije University Brussels, 2007.

38. Yamaguchi, J., Nishino, D., And Takanishi, A. Realization of dynamic biped walking varying joint stiffness using antagonistic driven joints. In Proc. of the IEEE Conf. on Robotics and Automation (1998), pp. 2022-2029.

39. Yamaguchi, J., And TAKAnishi, A. Development of a biped walking robot having antagonistic driven joints using nonlinear spring mechanism. In Proc. of the IEEE Conf. on Robotics and Automation (1997), pp. 185-192.

40. Zajac, F. E., Wicke, R. W., and Levine, W. S. Dependance of jumping performance on muscle properties when humans use only calf muscles for propulsion. Computer Methods in Biomechanics and Biomedical Engineering 17, 7 (1984), 513-523. 
Appendix: Expressions for matrices $D(\alpha), C(\alpha)$ and $E$ 
Symmetrical Matrix $D(\alpha)$ :

$d_{11}=I_{1}+m_{1} s_{1}^{2}+\left(m_{2}+m_{3}\right) l_{1}^{2}$,

$d_{12}=\left(m_{2} s_{2}+m_{3} l_{2}\right) l_{1} \cos \left(\alpha_{1}-\alpha_{2}\right)$,

$d_{13}=m_{3} l_{1} s_{3} \cos \left(\alpha_{1}-\alpha_{3}\right)$

$d_{14}=-\left(m_{1} s_{1}+m_{2} l_{1}+m_{3} l_{1}\right) \cos \alpha_{1}$

$d_{15}=-\left(m_{1} s_{1}+m_{2} l_{1}+m_{3} l_{1}\right) \sin \alpha_{1}$,

$d_{22}=I_{2}+m_{2} s_{2}^{2}+m_{3} l_{2}^{2}$,

$d_{23}=m_{3} l_{2} s_{3} \cos \left(\alpha_{2}-\alpha_{3}\right)$,

$d_{24}=-\left(m_{2} s_{2}+m_{3} l_{2}\right) \cos \alpha_{2}, d_{25}=-\left(m_{2} s_{2}+m_{3} l_{2}\right) \sin \alpha_{2}$,

$d_{33}=I_{3}+m_{3} s_{3}^{2}$,

$d_{34}=-m_{3} s_{3} \cos \alpha_{3}$,

$d_{35}=-m_{3} s_{3} \sin \alpha_{3}$,

$d_{44}=d_{55}=m_{1}+m_{2}+m_{3}$,

$d_{45}=0$,

$d_{i j}=d_{j i}, i \neq j$

Matrix $C(\alpha)$ :

\begin{tabular}{|c|c|c|c|}
\hline 0 & $\left(m_{2} s_{2}+m_{3} l_{2}\right) l_{1} \sin \left(\alpha_{1}-\alpha_{2}\right)$ & $m_{3} l_{1} s_{3} \sin \left(\alpha_{1}-\alpha_{3}\right)$ & $\begin{array}{ll}0 & 0\end{array}$ \\
\hline$c_{21}$ & 0 & $m_{3} l_{2} s_{3} \sin \left(\alpha_{2}-\alpha_{3}\right)$ & $0 \quad 0$ \\
\hline$c_{31}$ & $c_{32}$ & 0 & $0 \quad 0$ \\
\hline$\left(m_{1} s_{1}+m_{2} l_{1}+m_{3} l_{1}\right) \sin \alpha_{1}$ & $\left(m_{2} s_{2}+m_{3} l_{2}\right) \sin \alpha_{2}$ & $m_{3} s_{3} \sin \alpha_{3}$ & $\begin{array}{ll}0 & 0\end{array}$ \\
\hline$-\left(m_{1} s_{1}+m_{2} l_{1}+m_{3} l_{1}\right) \cos \alpha_{1}$ & $-\left(m_{2} s_{2}+m_{3} l_{2}\right) \cos \alpha_{2}$ & $-m_{3} s_{3} \cos \alpha_{3}$ & 0 \\
\hline
\end{tabular}

$c_{21}=-c_{12}, c_{31}=-c_{13}$ and $c_{32}=-c_{23}$ 
Diagonal constant matrix $E$ :

$\left[\begin{array}{ccccc}-\left(m_{1} s_{1}+m_{2} l_{1}+m_{3} l_{1}\right) & 0 & 0 & 0 & 0 \\ 0 & -\left(m_{2} s_{2}+m_{3} l_{2}\right) & 0 & 0 & 0 \\ 0 & 0 & -m_{3} s_{3} & 0 & 0 \\ 0 & 0 & 0 & 0 & 0 \\ 0 & 0 & 0 & 0 & m_{1}+m_{2}+m_{3}\end{array}\right]$

